\title{
Continuum Modeling
}

National Cancer Institute

\section{Source}

National Cancer Institute. Continuum Modeling. NCI Thesaurus. Code C75361.

The mathematical practice of applying a model to continuous data which has a potentially infinite number, and divisibility, of attributes. 